\title{
Acute Myeloid Leukemia in Children
}

\author{
Brenda E. S. Gibson, Martin G. Sauer, \\ and Persis Amrolia
}

\subsection{Introduction}

The outcome for children with acute myeloid leukemia (AML) has serially improved over the past three decades with an overall survival (OS) of $70-75 \%$ and event-free survival (EFS) of $60-65 \%$ widely reported. Much of this improvement is due to better supportive care, optimization of intensity of treatment including employment of Haematopoietic Stem Cell Transplantation (HSCT) in 1st complete remission (CR1) and better salvage in 2nd complete remission (CR2).

Whilst the majority of children ( $>90 \%)$ achieve $\mathrm{CR}$, the relapse rate (RR) in CR1 remains unacceptably high at $30-35 \%$, albeit varying by risk group. This global relapse risk has not improved significantly over the past three decades, and relapse remains the commonest cause of death.

B. E. S. Gibson $(\bowtie)$

Haematology Department, Royal Hospital for Children, University of Glasgow, Glasgow, UK e-mail: brenda.gibson@ggc.scot.nhs.uk

M. G. Sauer

Pediatric Hematology, Oncology and Blood Cell

Transplantation, Medizinische Hochschule Hannover, Hannover, Germany

P. Amrolia

Department of Bone Marrow Transplant, Great

Ormond St Children's Hospital, London, UK
HSCT, compared to chemotherapy as consolidation treatment, reduces the relapse risk in $\mathrm{CR} 1$ in all risk groups. However, this reduction in relapse risk has not always translated into an improvement in OS due to the treatment related mortality (TRM). The challenge is to identify children with a relapse risk in CR1, which is sufficiently high, to absorb the TRM and balance the risk in favour of HSCT. It is particularly important to establish the benefit of HSCT across all risk groups and within rare subtypes of AML associated with a poor outcome when treated with chemotherapy alone and not to assume that these children will benefit from HSCT. This will require evaluation by clinical trials which in turn will require international collaboration. Particularly worthy of consideration when weighing the benefits of transplantation in children are the associated late effects.

It is accepted that HSCT offers children with relapsed AML, who achieve a CR2, their only chance of long-term survival, and that some children with relapsed/refractory disease may benefit from HSCT.

\subsection{Prognostic Factors and Indications}

\subsubsection{First Complete Remission}

Consolidation therapy with allogeneic HSCT in CR1 of paediatric AML has been shown 
consistently to reduce the relapse risk through a GVL effect, which is stronger in AML than ALL.

Historically, HSCT trials employed a biological randomisation with children who had a matched sibling donor (MSD) receiving a HSCT as consolidation therapy and those without a MSD receiving consolidation chemotherapy. Improvements in HLA typing and donor selection have made HSCT an option for the majority of patients. Historical trials reported a reduction in RR which was counterbalanced by an increased TRM and better salvage for those who received chemotherapy only in CR 1 (Stevens et al. 1998; Woods et al. 2001; Lie et al. 2003). However, delaying transplant to CR2 carries risk. The mortality rate for reinduction after relapse is high, and those patients who do not achieve CR2 may be denied the opportunity of HSCT.

The criteria for transplanting patients have evolved from transplanting patients irrespective of risk group, to transplanting all patients other than those with good-risk cytogenetics (about $80 \%$ of all patients), to the current practice of restricting transplant to those with poor-risk (PR) cytogenetics (about $30 \%$ of all patients). These are the patients believed to be at the highest risk of relapse and therefore those most likely to benefit from HSCT in CR1 in an era of low TRM. There is no universal agreement on how high-risk (HR) disease should be defined. Different criteria have been, and continue to be, used by different national groups to define high risk. A combination of cytogenetics/molecular aberrations, which are currently considered to be the strongest indicator of outcome, and the presence of minimal residual disease (MRD), which may be assessed by morphology, flow cytometry or RT-PCR assessments of fusion transcripts, is evolving.

The percentage increase in disease free survival (DFS) or decrease in cumulative incidence of relapse (CIR) which would support HSCT as the best option in CR1 is undefined. This has been set at $10 \%$ in adults (Cornelissen et al. 2012), but the improved salvage after relapse and greater toll from late effects in children suggests that the bar should be set higher.

\subsubsection{Cytogenetics}

The cytogenetic abnormalities most commonly considered indicative of high risk of relapse include monosomy 7 , monosomy 5/5q-, abnormal 12p, $\operatorname{inv}(3) / t(3 ; 3) / a b n$ (3q), CBFA2T3GLIS2, $\mathrm{t}(4 ; 11), \mathrm{t}(5 ; 11), \mathrm{t}(6 ; 11), \mathrm{t}(10 ; 11), \mathrm{t}(6 ; 9)$ ,t $(9 ; 22), \mathrm{t}(7 ; 12), \mathrm{t}(11 ; 17), \mathrm{t}(8 ; 16), \mathrm{t}(3 ; 5)$ and complex karyotype. There is not complete consensus between national groups, and, in particular, not all agree that abnormalities of $3 \mathrm{q}, \mathrm{t}(6 ; 11)$ and complex (4 or more) are poor risk. Most consider a FLT3-ITD mutation (approximately $12 \%$ of children with AML) to be HR. However, some groups require the absence of good-risk cytogenetics, whilst other groups restrict this to FLT3 ITD-WT1 mutations or base the risk on the allelic ratio $(>0.4)$. The development of next-generation FLT3 inhibitors may challenge the role of HSCT in FLT3-ITD-mutated patients. A number of more recently recognised poor-risk cytogenetic abnormalities are cryptic, and it is expected that more cryptic abnormalities will be identified and that the list of poor-risk cytogenetic abnormalities may change with time. Currently poor-risk cytogenetics comprise about $25-30 \%$ of all AML in children.

An OS in excess of $70 \%$ is reported for HR patients after HSCT, although the definition of HR is not uniform. However, a combined COG and CIBMTR review of 233 children with AML between 1989 and 2006 with HR cytogenetics $(-7,7 q-,-5,5 q-$, abn 3q, t (6; 9), complex karyotype) reported no benefit for HSCT over chemotherapy. 123 children received chemotherapy, 55 a matched related donor (MRD) HSCT and 55 an unrelated donor (URD) HSCT. The 5-year OS from the time of consolidation or conditioning was similar: chemotherapy $43 \%$, MRD $46 \%$ and URD 50\% ( $p=0.99)$.

The pattern of failure differed: CIR at 5 years $61 \%$ vs. $51 \%$ vs. $30 \%$ for chemotherapy, MRD and URD, respectively $(p<0.001)$, and TRM $7 \%, 13 \%$ and $23 \%$, respectively $(p=0.005)$. HR was defined by cytogenetics alone and did not include FLT3 mutational or MRD status (Kelly et al. 2014). The benefit for HSCT in a number of poor-risk cytogenetic subgroups has not been proven and must not be assumed. 
Whilst HSCT is generally directed at patients defined as HR by cytogenetic abnormalities, a meta-analysis of MRC and POG trials of HSCT vs. chemotherapy showed that only intermediate-risk patients, where risk was defined by cytogenetics and poor morphological response to treatment, benefited from transplant (OS 61\% vs. 51\%). There was no advantage for HSCT in patients with poor-risk cytogenetics; however there were too few patients in the poor-risk group to make firm conclusions (Horan et al. 2008).

\subsubsection{Minimal Residual Disease Assessment (See Chap. 57)}

MRD is variably employed in risk stratification to direct patients to HSCT. It has been shown to be strongly predictive of outcome, and whilst it is commonly used to intensify treatment in poor responders, it may equally identify those with poor-risk cytogenetic aberrations who have a favourable early response and may not require HSCT. Currently multidimensional flow (MDF) cytometry is most commonly employed either by measuring leukemia aberrant immunophenotype (LAIP) or a "different from normal" phenotype. The discretionary level is $0.1 \%$, and the most commonly used time point is post course 2 . Alternatively, discretionary levels of $0.1 \%$ or $1 \%$ post course 1 are used by some groups to guide patients to HSCT in CR1. About $20 \%$ of patients with a MRD level $<0.1 \%$ after course 1 will relapse, which implies that genetic aberrations may influence relapse more than MRD. Similarly, $30 \%$ of patients with a MRD level of $>0.1 \%$ after course 1 will remain in remission. MDF cytometry is sensitive to a level of $0.1-0.01 \%$ and applicable in $90 \%$ of patients with AML.

Molecular MRD may be more sensitive and informative but data is limited. It is not commonly used outwith acute promyelocytic leukemia (APL), but our understanding and employment of molecular MRD may change with experience. MRD assessment by reverse transcription PCR (RT-PCR) for fusion transcripts has a sensitivity level of $0.01-001 \%$ and is applicable in approximately $50 \%$ of patients. The generally accepted discriminatory level is a greater than three-log reduction in transcript levels.
The relative significance of cytogenetics/ molecular aberrations and MRD status may evolve. Digital PCR and next-generation sequencing may be more sensitive but remain in the research arena. Similarly, leukemia stem cell monitoring may be more informative (Schuurhuis et al. 2018).

The main benefit of measuring MRD by any methodology is that it may allow the tailoring of the intensity of treatment. Thirty one of 267 (12\%) children treated on NOPHO-AML 20024 were defined as poor responders- $15 \%$ blasts morphologically after course 1 or $5 \%$ blasts after course 2 . These patients had time-intensive chemotherapy followed by HSCT in 25 of 31 with a donor. The 3-year probability of survival for these HR patients was $70 \%$. Patients classified as intermediate risk (defined as $5-14.9 \%$ blasts after course 1) had a significantly inferior EFS compared to HR patients. Both groups had timeintensive chemotherapy, but only HR patients proceeded to HSCT (Wareham et al. 2013; Abrahamsson et al. 2011).

The level of MRD after course 1 of chemotherapy in children treated on AIEOP 2002/01 correlated with outcome. At 8 years the outcomes for the 125 children in morphological remission with MRD level post course 1 of $<0.1 \%$ vs. $\geq 0.1 \%$ was DFS $73.1 \%$ vs. $35.2 \%$ ( $p<0.01$ ), OS $82.2 \%$ vs. $51.6 \%(p=0.0005)$ and CIR $23.5 \%$ vs. $62.8 \%(p=0.0005)$. Post course 2 , the outcomes at 8 years for MRD of $<0.1 \%$ vs. $\geq 0.1 \%$ was DFS $68.4 \%$ vs. $21.9 \%(p<0.01)$, OS $77.1 \%$ vs. $55.5 \%(p=0.0275)$ and CIR $31.6 \%$ vs. $73.9 \%$ $(p=0.00078)$.

Thirty-six patients had a MRD level of $\geq 0.1 \%$ at the end of course 1:13 achieved a MRD $<0.1 \%$ after course 2 , and their DFS was $45.4 \%$ vs. 22.8 $\%$ for patients with persisting MRD $\geq 0.1 \%$ $(p=0.037)$. Therefore, patients who achieve a level of MRD $<0.1 \%$ after course 2 but who were MRD positive $(>0.1 \%)$ after course 1 remain at higher risk of relapse and have a poorer outcome compared to those who are MRD negative after course 1 . This suggests that not only clearance of MRD but additional effective treatment is required to improve outcome (Buldini et al. 2017; Loken et al. 2012). 
St Jude's AML 02 study showed no difference in OS between HR patients undergoing HSCT compared to those who received chemotherapy. When the analysis was restricted to HR patients defined by MRD $>1 \%$ after induction 1 , the OS for HSCT was $43 \%$ vs. $23 \%$ for chemotherapy: $p=0.14$ (Rubnitz et al. 2010). Whilst the difference was not statistically significant, this may have been limited by low patient numbers.

There is no advantage for HSCT in CR1 for patients with good-risk cytogenetics- $\mathrm{t}(8 ; 21)$, inv (16), normal karyotype with NPM1 and normal karyotype with biallelic CEBPA. Some groups include $\mathrm{t}(1 ; 11)$ (q21; q23) in the goodrisk cytogenetic group.

The benefit of HSCT in CR1 for patients with intermediate-risk cytogenetics is less clear, and these may be the patients without PR cytogenetics but with a poor early response to chemotherapy in whom MRD can identify those at high risk of relapse. There is no role for HSCT in CR1 of APL or DS AML. HSCT for patients with Fanconi anaemia and MDS/AML and those with JMML are discussed elsewhere.

\subsubsection{Second Complete Remission}

Patients with relapsed AML have a dismal prognosis with chemotherapy alone, and it is generally accepted that they should proceed to transplant in CR2. The chance of achieving a second CR after relapse is dependent on the length of CR1: CR1 <1 year vs. CR >1 year is $50 \%$ vs. $75 \%$ with an overall CR rate of $60 \%$, OS for CR $<1$ year $26 \%$ vs. $45 \% \mathrm{CR}>1$ year, $p<0.001$ (Kaspers et al. 2013). Prognostically significant are the time to relapse, cytogenetics, no HSCT in CR1 and the speed of response to reinduction. Cytogenetics are strong prognostic indicators in relapse as in de novo disease with patients with CBF leukemias fairing the best: $\mathrm{CBF}$ leukemias vs. others-OS $67 \%$ vs. $31 \%, p<0.001$.

\subsubsection{Refractory Disease}

It has long been accepted that a poor morphological response $>5 \%$ blasts at day 15 or resistant dis- ease after course 1 or 2 has a poor outcome with chemotherapy alone. If CR cannot be achieved, the outlook is poor, but aggressive chemotherapy followed by HSCT may benefit some patients. Residual disease/MRD positivity pre-HSCT increases the risk of relapse post-HSCT, but the susceptibility of AML to GVL does not preclude transplant. MRD status just prior to HSCT is an important prognostic indicator.

A small study reported a 5-year OS of $80.4 \%$ for children with $<0.01 \% \operatorname{MRD}(n=27), 66.7 \%$ for those with $0.01-5 \%$ MRD $(n=9)$ and $58.3 \%$ for those with $>5 \%$ MRD (Leung et al. 2012). It is not clear what level of disease should preclude HSCT.

The role of transplant in CR1, CR2 and refractory disease may change with time if new effective chemotherapy agents become available.

\subsection{Conditioning Regimens}

No advantage has been shown for total body irradiation (TBI) in AML and chemotherapy-only regimens should be used. Adult data from the CIBMTR demonstrated improved non relapse mortality (NRM), OS and DFS in patients with AML transplanted using IV Busulfan $(\mathrm{Bu})$ with therapeutic drug monitoring (TDM) compared with TBI (Copelan et al. 2013). Myeloablative conditioning (MAC) regimens are most commonly used, but a number of reduced toxicity conditioning (RTC) regimens are being tested. There is no proven "best" chemotherapy conditioning regimen, though $\mathrm{MAC}$ regimens with $\mathrm{Bu}$ and cyclophosphamide (Cy) with TDM of $\mathrm{Bu}$ levels are currently the standard of care. A retrospective EBMT study of $\mathrm{Bu}, \mathrm{Cy}$ and Melphalan (Mel) (enhanced MAC) in paediatric AML in CR1 suggested improved RR and Leukemia free survival (LFS) compared with $\mathrm{BuCy}$, but the majority of patients receiving $\mathrm{BuCy}$ on this study did not undergo TDM (Lucchini et al. 2017). Moreover, whilst this regimen is well tolerated in children under the age of 12 years, it is associated with non-acceptable TRM rates between 20 and $30 \%$ in teenagers and should therefore be avoided or used with caution in this group (Sauer et al. 2017). There is an increasing body of experience with MAC $\mathrm{Bu}$ and 
Fludarabine (Flu), which is well tolerated, but no randomised comparisons are available to determine relative anti-leukaemic activity of BuFlu vs BuCy (Harris et al. 2018). Replacing Bu with Treosulfan (Treo) to reduce toxicity whilst maintaining efficacy is being tested and given in combination with $\mathrm{Cy}$ (TreoCy) or with Flu and Thiotepa (FTT). The choice of conditioning regimen is a balance between efficacy and toxicity. Comorbidity; pretreatment with drugs which may contribute to toxicity, i.e. gemtuzumab and VOD/SOS, age and HLA disparity may influence the choice of conditioning regimen. Comorbidity or heavy previous treatment may indicate a reduced intensity conditioning (RIC) with BuFlu or FluMel. Targeted Bu levels will differ between MAC, RTC and RIC. Patient toxicities may suggest avoidance of specific agents. Newer regimens which include clofarabine are also being tested.

A retrospective comparison of RIC (39) vs. MAC (141) in matched patients has reported no difference in a-GVHD, c-GVHD, TRM, LFS and OS. The OS was $45 \%$ vs. $48 \%, p=0.99$; RR $39 \%$ vs. $39 \%, p=0.95$; and TRM $16 \%$ vs. $16 \%$, $p=0.73$. However, about $50 \%$ of MAC used TBI, whilst BuCy was the commonest chemotherapy regimen. Patients who received a RIC had had more pre-HSCT morbidity. The performance score influenced OS, LFS and CIR (Bitan et al. 2014). The current paediatric AML protocol MyeChild01 is prospectively comparing MAC $\mathrm{BuCy}$ with a reduced toxicity BuFlu regimen.

\subsection{Donor Selection Hierarchy and Stem Cell Source}

\subsubsection{Autologous HSCT}

There is no evidence from a number of studies and meta-analysis that auto-HSCT is superior to intensive chemotherapy as consolidation therapy and it is now not employed. AML 2002/01 study reported a DFS of $73 \%$ for allo-HSCT in HR patients compared to $63 \%$ for auto-HSCT, p-ns. The CIR was $17 \%$ vs. $28 \%$ in favour of allo-HSCT, $p=0.043$. There was no difference in TRM at $7 \%$ for both groups at 8 years (Locatelli et al. 2015).

\subsubsection{Allogeneic HSCT}

The choice of donor for allo-HSCT is based on HLA compatibility and CMV status. Outcomes are similar for MSD and well matched unrelated donors (UDs). The degree of mismatch which is acceptable depends on the risk of relapse and CR status. Mismatched unrelated donor (MMUD) or cords and haplo-HSCT are generally reserved for very HR disease or early relapses.

Patients and their siblings should be tissuetyped at diagnosis. In the absence of a HLA matched family donor (MFD), an URD and cord blood unit (CBU) search should be initiated as soon as possible after induction course 1 for patients with intermediate or PR cytogenetics. Donors should be selected using the selection hierarchy of the national group. Medium-/highresolution typing is required for adult URD (HLA A, B, C, HLA-DRB 1 and HLA-DQB1) and unrelated cords (HLA A, B, C and DR loci).

The risk of relapse does not just direct the need for transplant but the HLA discrepancy which is acceptable. MFD or well-MUD should be identified for $\mathrm{CR} 1$ patients, whilst mismatched donors (8/10 MMUD), cords (4/8 MMUCB) or haplo-HSCT should be reserved for very highrisk disease, CR2 or refractory disease.

For family/unrelated donors, BM is the preferred stem cell source, but the use of PBSC is permissible and is more commonly used. The use of PBSC from mismatched donors should be avoided wherever possible.

In the UK, serotherapy is only given to patients transplanted from unrelated donors, 9/10 mismatched family donors or $5 / 8$ matched cords blood units, but not to patients receiving grafts from matched family donors or 6-8/8 unrelated cord blood units. Other European groups only employ T-cell depletion in mismatched donors (MMUD).

\subsection{GVHD Prophylaxis}

All patients should receive immunosuppression (IS) with ciclosporin (CSA). Most, but not all, national groups add short-course methotrexate (MTX) for all patients. Patients receiving grafts 
from a mismatched donor or those in whom the stem cell source is PBSC or unrelated cord blood should receive prophylaxis in addition to CSA with either mycophenolate mofetil (MMF) or short-course MTX. In adult AML, increased exposure to CSA was associated with increased relapse and decreased survival (Craddock et al. 2010), supporting early withdrawal of IS where possible. In the absence of GVHD, MMF can be stopped at day 28 post transplant, and CSA tailed over 4-6 weeks from day 60 (MFD), day 100 (MUD) or earlier if mixed chimerism is detected in the whole blood.

\subsection{Donor Lymphocyte Infusions (DLI)}

The evidence of benefit for DLI is weak. Rettinger et al. investigated the use of pre-emptive immunotherapy with reduction of IS and low-dose DLI in patients with paediatric AML developing mixed chimerism (MC) after HSCT for AML; 6/13 patients with MC who received immunotherapy remained in long-term $\mathrm{CR}$, whereas all 7 patients with MC who did not receive immunotherapy relapsed (Rettinger et al. 2017). Based on these limited data, our practice is to use preemptive immunotherapy in patients with confirmed MC (defined as $>1 \%$ autologous cells in the whole blood on two occasions 1 week apart) without active acute GVHD > Grade 1 or chronic GVHD in the first-year post transplant. If patients are still receiving IS, this should be discontinued and chimerism reassessed a month later. In patients already off IS, chimerism should be reassessed a month off IS. If mixed chimerism persists, DLI should be given to recipients of MFD or MUD. DLI is not recommended in the context of 9/10 mismatched donor HSCT. The DLI cell dose administered is dependent on the donor source and the timing post transplant. In the future, the use of pre-emptive DLI is likely to be based on detection of flow or molecular MRD in the bone marrow.

\subsection{Management of Relapse Post transplant}

For selected patients who relapse late ( $>1$ year) post first HSCT and respond to reinduction chemotherapy, a second transplant may be curative with survival rates of $24-35 \%$ reported (Yaniv et al. 2018; Uden et al. 2017). DLI is of limited efficacy in frank relapse post transplant except if a further remission can be achieved (Schmid et al. 2007; Kolb et al. 1995).

Interestingly, CR has been seen in cutaneous (but not bone marrow) relapse of AML post transplant with the checkpoint inhibitor ipilimumab (Davids et al. 2016). Novel agents targeting specific pathways, e.g. FLT 3 inhibition, have met with limited success to date. Treatment options for patients who relapse early after transplant are challenging, and at present, for the majority of such patients, we recommend symptom care or enrolment in a clinical trial. Antibody-drug conjugates, bispecific T-cell-engaging antibodies and chimeric antigen receptor (CAR) T cells under development may be tested in such patients in the future (Table 70.1).

Table 70.1 Donor and source for HSCT: the hierarchy of the UK

\begin{tabular}{|c|c|c|c|}
\hline Choice & $\begin{array}{l}\text { Family } \\
\text { donor }\end{array}$ & $\begin{array}{l}\text { Unrelated } \\
\text { donor }\end{array}$ & Unrelated cord \\
\hline $1 \mathrm{st}$ & $\begin{array}{l}\text { MFD (BM, } \\
\text { PBSC, } \\
\text { CB) }\end{array}$ & & \\
\hline 2nd & & $\begin{array}{l}\text { 10/10 MUD } \\
\text { 9/10 1DQ } \\
\text { MMUD }\end{array}$ & $\begin{array}{l}8 / 8 \text { MUCB (total } \\
\text { nucleated cell } \\
\left(\mathrm{TNC}>3 \times 10^{7} / \mathrm{kg}\right)\end{array}$ \\
\hline $3 \mathrm{rd}$ & $\begin{array}{l}9 / 10 \\
\text { MMFD }\end{array}$ & $\begin{array}{l}\text { 9/10 (other) } \\
\text { MMUD }\end{array}$ & $\begin{array}{l}5-7 / 8 \text { MMUCB } \\
\left(\mathrm{TNC}>3 \times 10^{7} / \mathrm{kg}\right)^{\mathrm{a}}\end{array}$ \\
\hline
\end{tabular}

$M F D$ matched family donor, $M U D$ matched unrelated donor, $M M U D$ mismatched unrelated donor, $M U C B$ matched unrelated cord blood, $M M F D$ mismatched family donor, $M M U C B$ mismatched unrelated cord blood aFor unrelated cord blood, a single cord is used if the cryopreserved TNC dose is $>3 \times 10^{7} / \mathrm{kg}$. If $<3 \times 10^{7} / \mathrm{kg}$, a double cord transplant is preferred 


\section{Key Points}

- There is increasing evidence that patients with cytogenetic or molecular high-risk features may benefit from HSCT in CR1. About $30 \%$ of children fall into this risk group. A TRM below $10 \%$ should be achievable.

- A matched family or unrelated donor is considered the optimal donors and the bone marrow the preferred stem cell source. Mismatched donors may be considered appropriate for patients with poorly responding disease.

- Children who achieve CR2 after first relapse have a bleak prognosis without HSCT.

- MAC, TBI-free, conditioning is recommended for patients transplanted in CR1 and CR2, and to date the standard regimen had been BuCy.

- Novel conditioning regimens incorporating TREO or clofarabine are being explored, and these need to be compared with $\mathrm{BuCy}$ in prospective, randomised studies.

- Relapse after HSCT in CR1 is associated with a very poor outcome and not curable without a second HSCT. TRM for second HSCTs exceeds $30 \%$ which might favour the use of a RIC in this setting. The prevention of relapse remains the major challenge.

\section{References}

Abrahamsson J, Forestier E, Heldrup J, et al. Responseguided induction therapy in pediatric acute myeloid leukemia with excellent remission rate. J Clin Oncol. 2011;29:310-5.

Bitan M, He W, Zhang MJ, et al. Transplantation for children with acute myeloid leukaemia: a comparison of outcomes with reduced intensity and myeloablative regimens. Blood. 2014;123:1615-20.
Buldini B, Rizzati F, Masetti R, et al. Prognostic significance of flow-cytometry evaluation of minimal residual disease in children with acute myeloid leukaemia treated according to the AIEOP-AML 2002/01 study protocol. Br J Haematol. 2017;177:116-26.

Copelan EA, Hamilton BK, Avalos B, et al. Better leukaemia-free and overall survival in AML in first remission following cyclophosphamide in combination with Busulfan compared with TBI. Blood. 2013; 122:3863-70.

Cornelissen JJ, Gratwohl A, Schlenk RF, et al. The European LeukaemiaNet AML Working Party consensus statement on allogeneic HSCT for patients with AML in remission: an integrated-risk adapted approach. Nat Rev Clin Oncol. 2012;9: 579-90.

Craddock C, Nagra S, Peniket A, et al. Factors predicting long-term survival after T-cell depleted reduced intensity allogeneic stem cell transplantation for acute myeloid leukaemia. Haematologica. 2010;95:989-95.

Davids MS, Kim HT, Bachireddy P, et al. Ipilimumab for patients with relapse after allogeneic transplantation. N Engl J Med. 2016;375:143-53.

Harris AC, Boelens JJ, Ahn KW, et al. Comparison of pediatric allogeneic transplant outcomes using myeloablative Busulfan with cyclophosphamide or fludarabine. Blood Adv. 2018;2:1198-206.

Horan JT, Alonzo TA, Lyman GH, et al. Impact of disease risk on efficacy of matched related bone marrow transplantation for pediatric acute myeloid leukaemia; the Children's Oncology Group. J Clin Oncol. 2008;26:5797-801.

Kaspers GL, Zimmermann M, Reinhardt D, et al. Improved outcome in pediatric relapsed acute myeloid leukaemia: results of a randomised trial on liposomal daunorubicin by the International BFM Study Group. J Clin Oncol. 2013;31:599-607.

Kelly MJ, Horan JT, Alonzo TA, et al. Comparable survival for pediatric acute myeloid leukaemia with poorrisk cytogenetics following chemotherapy, matched related donor or unrelated donor transplantation. Pediatr Blood Cancer. 2014;61:269-75.

Kolb HJ, Schattenberg A, Goldman JM, et al. Graftversus-leukemia effect of donor lymphocyte transfusions in marrow grafted patients. European Group for Blood and Marrow Transplantation Working Party Chronic Leukemia. Blood. 1995;86:2041-50.

Leung W, Pui CH, Coustan-Smith E, et al. Detectable minimal residual disease before hematopoietic cell transplantation is prognostic but does not preclude cure for children with very -high-risk leukaemia. Blood. 2012;120:468-72.

Lie SO, Abrahamsson J, Clausen N, et al. Treatment stratification based on initial in vivo response in acute myeloid leukaemia in children without Down's 
syndrome: results of NOPHO-AML trials. $\mathrm{Br} \mathrm{J}$ Haematol. 2003;122:217-25.

Locatelli F, Masetti R, Rondelli R, et al. Outcome of children with high -risk acute myeloid leukaemia given autologous or allogeneic hematopoietic cell transplantation in the AIEOP AML-2002/01 study. Bone Marrow Transplant. 2015;50:181-8.

Loken MR, Alonzo TA, Pardo L, et al. Residual disease detected by multidimensional flow cytometry signifies high relapse risk in patients with de novo acute myeloid leukaemia: a report from Children's Oncology Group. Blood. 2012;120:1581-8.

Lucchini G, Labopin M, Beohou E, et al. Impact of conditioning regimen on outcomes for children with acute myeloid leukaemia undergoing transplantation in first complete remission. an analysis on behalf of the Pediatric Disease Working Party of the European Group for Blood and Marrow Transplantation. Biol Blood Marrow Transplant. 2017;23:467-74.

Rettinger E, Merker M, Salzmann-Manrique E, et al. Preemptive immunotherapy for clearance of molecular disease in childhood acute lymphoblastic leukaemia after transplantation. Biol Blood Marrow Transplant. 2017;23:87-95.

Rubnitz JE, Inaba H, Dahl G, et al. Minimal residual disease - directed therapy for childhood acute myeloid leukaemia; results of the AML 02 multicentre trial. Lancet Oncol. 2010;11:543-52.

Sauer MG, Lang P, Albert M, et al. AML SCT-BFM 2007: results of the prospective hematopoietic stem cell transplantation (HSCT) trial of the BerlinFrankfurt-Münster (BFM) Study Group for children with very high risk acute myeloid leukemia. Blood. 2017;130(Suppl 1):599.

Schmid C, Labopin M, Nagler A, et al. Donor lymphocyte infusion in the treatment of first hematological relapse after allogeneic stem-cell transplantation in adults with acute myeloid leukaemia: a retrospective risk factors analysis and comparison with other strategies by the EBMT Acute Leukaemia Working Party. Clin Oncol. 2007;25:4938-45.

Schuurhuis GJ, Heuser M, Freeman S, et al. Minimal/ measurable residual disease in AML: a consensus document from the European leukemiaNet MRD working party. Blood. 2018;131:1275-91.

Stevens RF, Hann IM, Wheatley K, Gray RG. Marked improvements in outcome with chemotherapy alone in paediatric acute myeloid leukaemia: results of the United Kingdom Medical Research Council's 10th AML trial. MRC Childhood Leukaemia Working Party. Br J Haematol. 1998;101:130-40.

Uden T, Bertaina A, Abrahamsson J, et al. Outcome of children relapsing after first allogeneic hematopoietic stem cell transplantation for pediatric acute myeloid leukemia: a retrospective I-BFM analysis of 336 children between 2005 and 2016. Blood. 2017;130(Suppl 1):272.

Wareham NE, Heilmann C, Abrahamsson J, et al. Outcome of poor response paediatric AML using early SCT. Eur J Haematol. 2013;90:187-94.

Woods WG, Neudorf S, Gold S, et al. A comparison of allogeneic bone marrow transplantation, autologous bone marrow transplantation, and aggressive chemotherapy in children with acute myeloid leukaemia in remission. Blood. 2001;97:56-62.

Yaniv I, Krauss AC, Beohou E, et al. Second hematopoietic stem cell transplantation for post-transplantation relapsed acute leukaemia in children: a retrospective EBMT-PDWP Study. Biol Blood Marrow Transplant. 2018;24(8):1629-42.

Open Access This chapter is licensed under the terms of the Creative Commons Attribution 4.0 International License (http://creativecommons.org/licenses/by/4.0/), which permits use, sharing, adaptation, distribution and reproduction in any medium or format, as long as you give appropriate credit to the original author(s) and the source, provide a link to the Creative Commons license and indicate if changes were made.

The images or other third party material in this chapter are included in the chapter's Creative Commons license, unless indicated otherwise in a credit line to the material. If material is not included in the chapter's Creative Commons license and your intended use is not permitted by statutory regulation or exceeds the permitted use, you will need to obtain permission directly from the copyright holder. 